\title{
miR-16 targets SALL4 to repress the proliferation and migration of gastric cancer
}

\author{
XUEFENG JIANG and ZHE WANG \\ Department of Gastroenterology, China-Japan Union Hospital, Jilin University, Changchun, Jilin 130000, P.R. China
}

Received January 29, 2018; Accepted June 8, 2018

DOI: $10.3892 / 01.2018 .8997$

\begin{abstract}
There is increasing evidence that microRNAs (miRNAs) play important roles in tumor progression and development by targeting different genes, including gastric cancer (GC). However, the role of miR-16 in GC is so far unclear. Herein, we examined the function and potential mechanism of miR-16 in GC. Reverse transcription-quantitative PCR found that miR-16 expression was prominently lower in GC tissues while SALL4 expression was frequently higher than normal tissues. Re-expression of miR-16 could suppress GC cell proliferation and migration by MTT and Transwell assay. We confirmed that miR-16 directly targeted SALL4 in regulating GC by luciferase assay. Knockdown of SALL4 inhibited cell proliferation and migration. Furthermore, SALL4 could counteract the inhibition-effect of miR-16 in GC. In conclusion, for the the first time we demonstrated that miR-16 played inhibitory effect through targeting SALL4 in $\mathrm{GC}$ cell proliferation and migration. Our study revealed that miR-16/SALL4 axis was critical in regulating the GC development, indicating a new prospect to regulate GC cell progression and development.
\end{abstract}

\section{Introduction}

Gastric cancer (GC) is one of the most common malignant diseases originating from the mucosal epithelium of the stomach. GC has high morbidity and mortality in China, which seriously affects the health of patients $(1,2)$. GC is more aggressive but is hard to find in early stage, so most GC patients are diagnosed at an advanced stage (3). Although clinically significant progress has been made in treatment, the clinical outcomes of patients with advanced GC have not had a significant impact. Therefore, studies to explore the underlying

Correspondence to: Dr Zhe Wang, Department of Gastroenterology, China-Japan Union Hospital, Jilin University, 126 Xiantai Street, Changchun, Jilin 130000, P.R. China

E-mail: wmeqef@163.com

Key words: miR-16, SALL4, repress, proliferation, migration, gastric cancer mechanisms of the GC development are necessary, as they could provide novel therapeutic targets for GC treatment (4).

Increasing evidence has been reported that microRNAs (miRNAs) could function as tumor inhibitors or tumor promoters in the GC development by targeting several mRNA genes, including proliferation, migration and invasion $(5,6)$. For example, Ahn et al (7) showed that miR-200 acted as an oncogene in modulating GC progression via inhibiting CDH1. However, miR-22 was proved to suppress GC metastasis and invasion via regulating MMP14 and Snail (8). So far, the miRNAs that were found to participate in GC development are still relatively limited, and their roles and potential mechanisms need to be further studied.

Many previous studies showed that miR-16 is involved in cell proliferation, invasion and metastasis of various cancers. miR-16 was proven to function as a tumor suppressor in regulating glioma cell proliferation, invasion and promoted apoptosis through targeting Wipl (9). A previous study also showed that the effect of miR-127 on non-small cell lung cancer proliferation was inhibition (10). In addition, one study stated that miR-127 acted as a tumor promoter in regulating of the progression of colorectal adenocarcinoma (11). However, there are very few studies on the biological mechanism of miR-127 in GC.

Sal-like protein 4 (SALL4) is a zincfinger transcription factor encoded by a member of the SALLgene family (12). Previous studies showed that the role SALL4 played in early embryo development, organ formation and the proliferation and pluripotency of embryonic stem cells was very important (13-16). Recently, SALL4 was shown to be involved in modulating various solid tumors. For instance, SALL4 expression was upregulated in liver, lung, breast and colorectal cancer (17-20). Furthermore, SALL4 could promote the migratory and invasive ability of breast cancer (21) and cell viability of endometrial cancer (22). Therefore, to deeply understand the mechanism of SALL4 in cancers would help researchers to find a new target for cancer diagnosis and treatment (23). A study recently reported that SALL4 promoted GC progression as an oncogene $(24,25)$. However, the biological role of SALL4 in $\mathrm{GC}$ regulated by miR-16 remains unclear.

Our study examined miR-16 in GC development and its biological mechanism in regulation of GC cell proliferation and migration. We found that miR-16 showed inhibitory effect in GC. miR-16 overexpression could suppress GC cell viability and migration and make SALL4 expression lower, while 


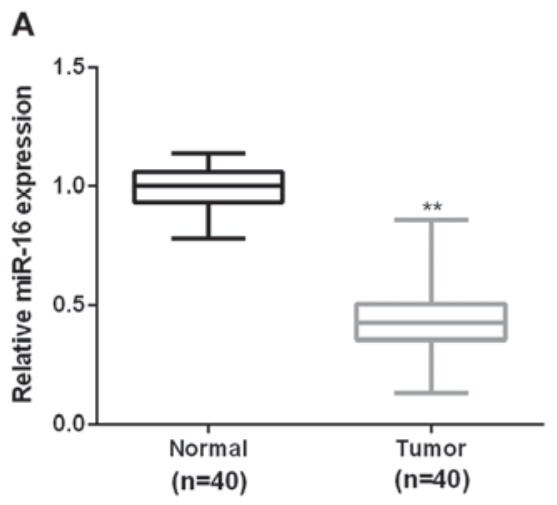

D

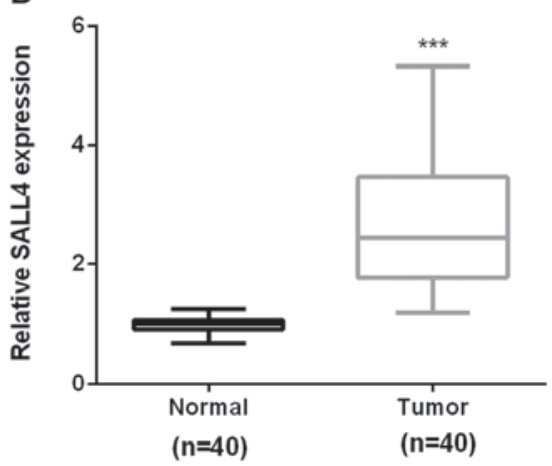

E

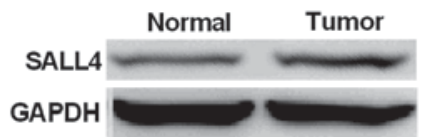

B

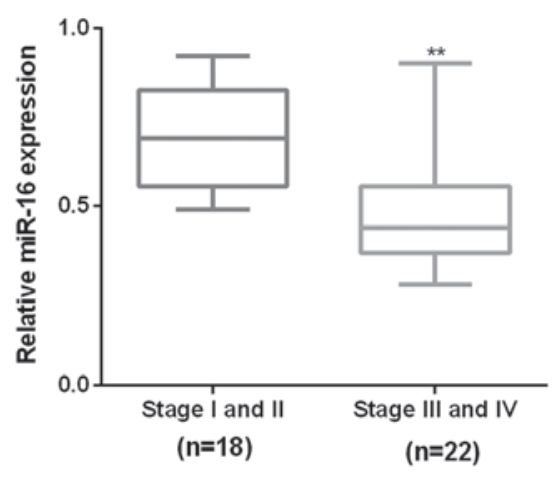

C

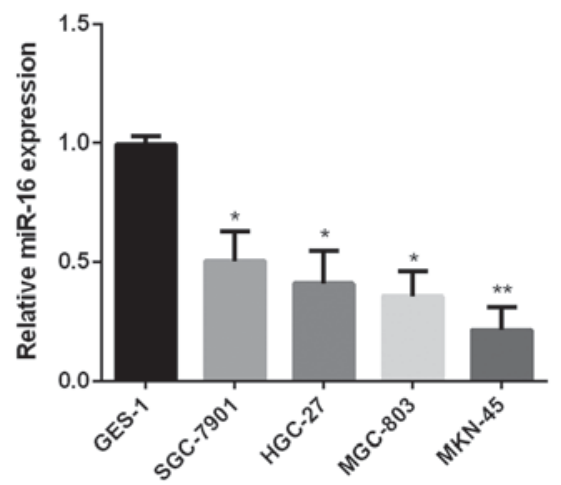

$\mathbf{F}$

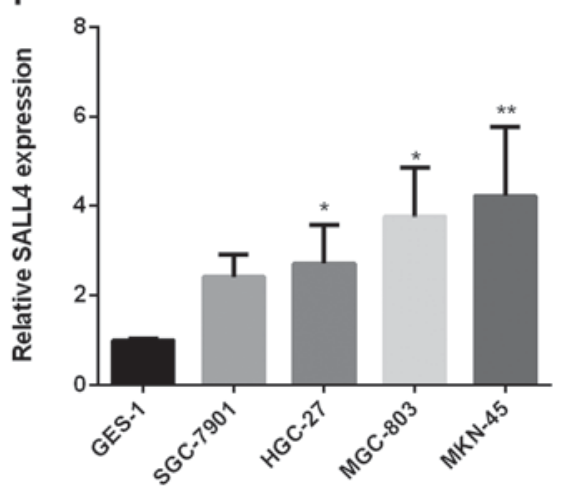

G

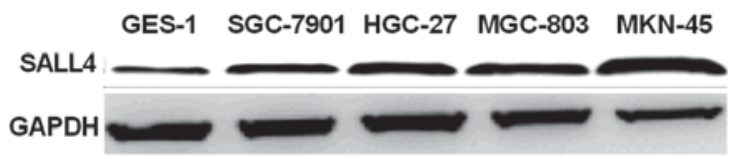

Figure 1. Decrease of miR-16 and increase of SALL4 in GC. (A) miR-16 expression in 40 paired GC tumor and normal tissues. (B) Differential expression of miR-16 in stage I/II and stage III/IV of GC tumor. (C) miR-16 expression in GC cells and normal GES-1 cells. (D and E) SALL4 mRNA and protein expression in GC tissues. (F and G) SALL4 mRNA and protein expression in GC cells and GES-1 cells. ${ }^{*} \mathrm{P}<0.05,{ }^{* * *} \mathrm{P}<0.01,{ }^{* * * *} \mathrm{P}<0.001$.

knockdown of miR-16 had the opposite effect. Furthermore, we demonstrated that the relationship between miR-16 and SALL4 expression was negatively correlated in GC tissues. Therefore, our results indicated that the miR-16/SALL4 axis provided a therapeutic target for treating GC.

\section{Materials and methods}

Samples and cell culture. Forty paired GC tissues and adjacent normal tissues were obtained from GC patients who underwent surgery at the China-Japan Union Hospital, Jilin University (Changchun, China). All tissue specimens were confirmed by pathological diagnoses and no patients received radiotherapy or chemotherapy before surgery. All corrected tissues were immediately frozen in $-80^{\circ} \mathrm{C}$ refrigerator. All contents about this study were approved by the Ethics Committee of China-Japan Union Hospital, Jilin University. Each GC patient involved in this study signed the informed consent.

The gastric epithelium cell line GES-1 and four GC cell lines (SGC-7901, HGC-27, MKN45 and MGC-803) were obtained from Shanghai Institute of Cell Biology of the Chinese Academy of Sciences. The cells were cultured in RPMI-1640 medium (Invitrogen; Thermo Fisher Scientific
Inc., Waltham, MA, USA) containing $10 \%$ fetal bovine serum, and then cultured in an incubator at $37^{\circ} \mathrm{C}$ under $5 \% \mathrm{CO}_{2}$.

Cell transfection. miR-16 mimic and inhibitor were provided by the company of GenePharma (Shanghai, China). miR-16 mimic and miR-16 inhibitor $(50 \mathrm{nM})$ were transfected into SGC-7901 and HGC-27 cells respectively in parallel to overexpress or suppress miR-16 and SALL4 small interfering RNA (siRNA) to silence SALL4. All the cells were plated in 24-well plates $24 \mathrm{~h}$ before transfection and the transfections were performed using Lipofectamine 3000 reagent (Invitrogen; Thermo Fisher Scientific Inc.) the next day. The transfected cells were divided into several groups.

RT- $q$ PCR assays. TRIzol reagent (Invitrogen; Thermo Fisher Scientific Inc.) was used to isolate total RNA from the GC tissues and cells. NanoDrop ND-1000 spectrophotometer (Thermo Fisher Scientific, Inc.), was used to quantify the RNA. The sequences of the primers were: miR-16, TAGCAG CACGTAAATATTGGCG (forward) and TGCGTGTCGTG GAGTC(reverse);forSALL4,TAGCCCTGCGTAGCCAGTTA (forward) and TCATGCTTAGTCCACTGTCTGT (reverse); for U6, GCTTCGGCAGCACATATACTA AAAT (forward) and CGCTTCACGAATTTGCGTGTCAT (reverse); for 
A

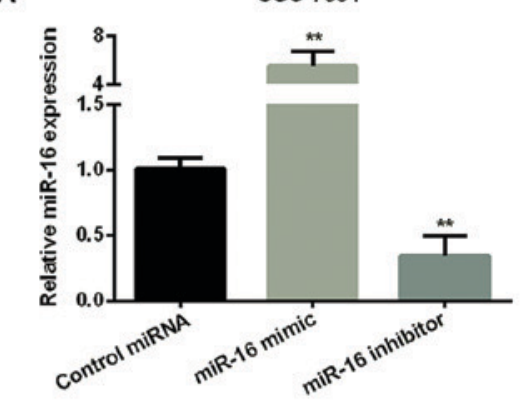

B

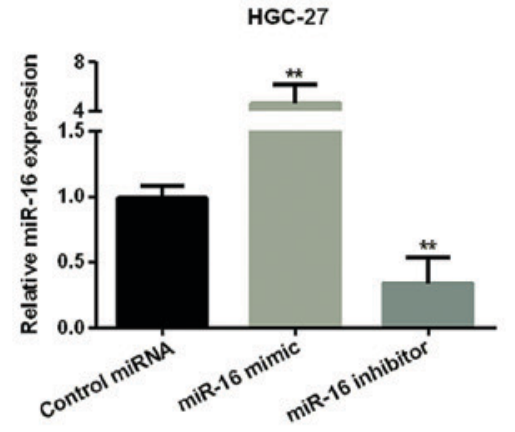

C

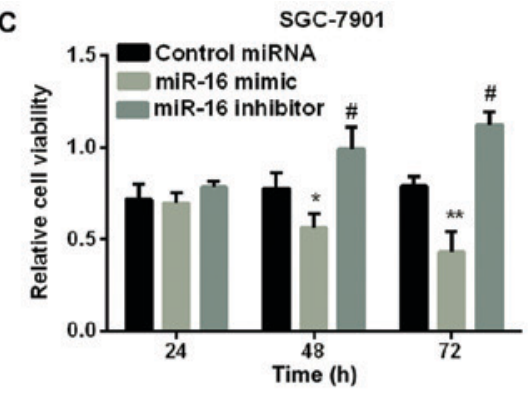

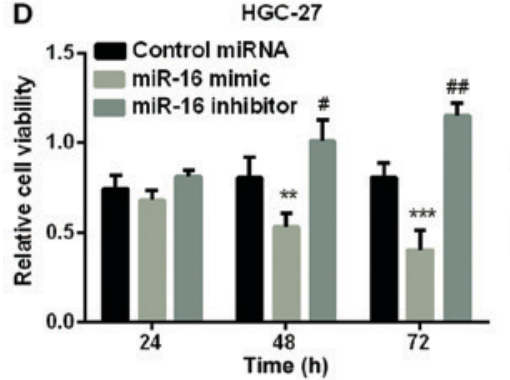

E

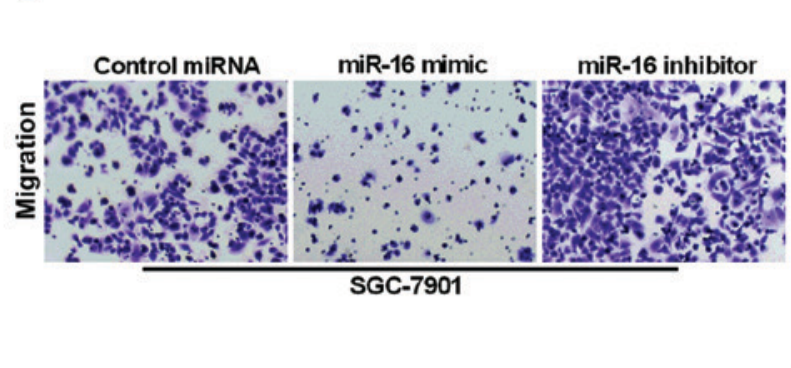

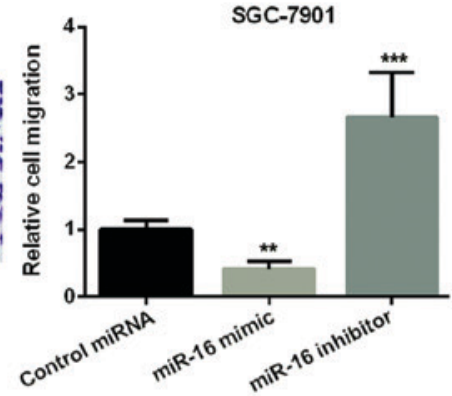

F

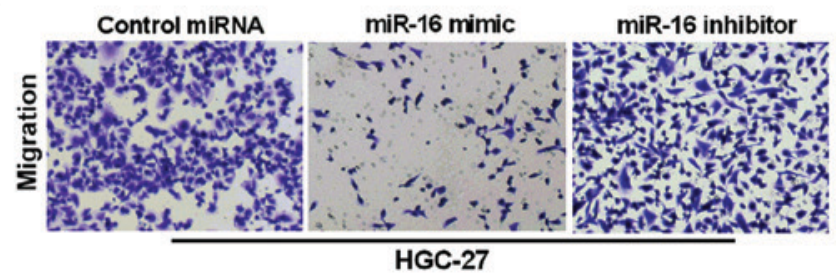

HGC-27

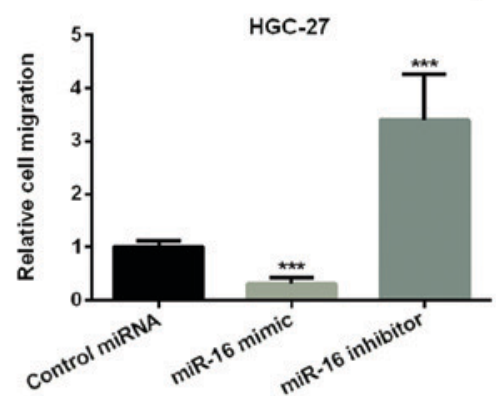

Figure 2. Abnormal expression of miR-16 affects the proliferation and migration ability of GC. (A and B) miR-16 expression increased observably in miR-16 mimic group, while decreased in miR-16 inhibitor group in both GC cell lines. (C and D) GC cell viability in the cell lines examined by MTT assays after overexpression or knockdown of miR-16. (E and F) GC cell migration in the cell lines examined by Transwell assays after overexpression or knockdown of miR-16. ${ }^{*} \mathrm{P}<0.05,{ }^{* *} \mathrm{P}<0.01,{ }^{* * *} \mathrm{P}<0.001 ;{ }^{*} \mathrm{P}<0.05,{ }^{\# \#} \mathrm{P}<0.01$

GAPDH, AGAAGGCTGGGGCTCATTTG (forward) and AGGGGCCATCCACAGTCTTC (reverse). U6 and GAPDH were used as internal controls. The $2^{-\Delta \Delta C q}$ method was used to detect the relative expression of miR-16 and SALL4 (26).

Cell proliferation assay. MTT assay was used to detect cell viability. SGC-7901 and HGC-27 cells were seeded and RPMI-1640 medium was subsequently added into 96-well plates and incubated for 24,48 , and $72 \mathrm{~h}$ at $37^{\circ} \mathrm{C}$ with $5 \%$ $\mathrm{CO}_{2}$. MTT solution was added to each well for incubation for $4 \mathrm{~h}$. After centrifugation, at room temperature, $1,000 \mathrm{x} \mathrm{g}$ for $10 \mathrm{~min}$, the culture medium was removed and DMSO (100 $\mu \mathrm{l})$ was added into the plates to dissolve the crystals. The absorbance value of each well was measured at the OD490 nm using enzyme-linked immunoassay.

Cell migration assay. Cell migratory ability was performed using Transwell assay. The Transwell chamber with $8 \mu \mathrm{m}$ pore size polycarbonic membrane (Costar; Corning Incorporated, Corning, NY, USA) was placed into the 24-well plates to separate the top and the lower chambers. GC cells $\left(1 \times 10^{5}\right)$ with different transfection were seeded into the top chamber, and RPMI-1640 medium containing 20\% fetal bovine serum was added into the lower chambers as an attractant and then incubated for $24 \mathrm{~h}$ at $37^{\circ} \mathrm{C}$. The cells in upper chambers subsequently migrated into the lower chamber. Then the migratory cells were stained with $0.1 \%$ crystal violet for $30 \mathrm{~min}$. Images of the migration cells were photographed under a microscope (SZ61; Olympus Corporation, Tokyo, Japan).

Western blot analysis. Total protein was extracted from the $\mathrm{GC}$ cells or tissues after transfection for $48 \mathrm{~h}$, and the protein concentration was measured. Then, $50 \mu \mathrm{g}$ protein samples in each group were subjected to $10 \%$ SDS-PAGE to separate the protein samples. Then, they were electrophoretically transferred to NC membrane (EMD Millipore, Billerica, MA, USA). Subsequently, skim milk (5-10\%) dissolved by $0.1 \%$ tris buffered saline with Tween-20 (TBST) was added to block the membranes for $2 \mathrm{~h}$ at room temperature. Firstly, the membranes were incubated with the primary antibody rabbit monoclonal anti-SALL4 (cat. no. 5850S; 1:1,000, Cell Signaling Technology, Inc., Danvers, MA, USA) at $4^{\circ} \mathrm{C}$ overnight, after washed with 1 xTBST (pH 7.4) three times later; the secondary antibodies goat anti-rabbit IgG-HRP 
A

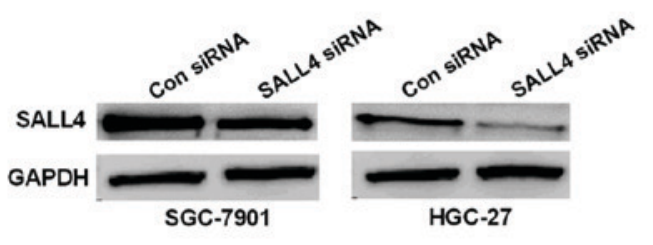

B

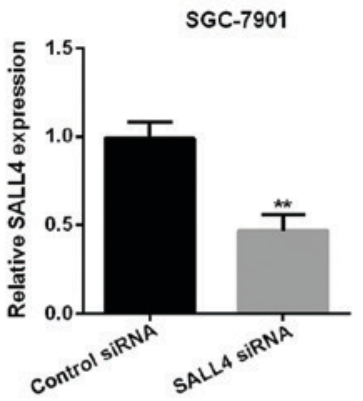

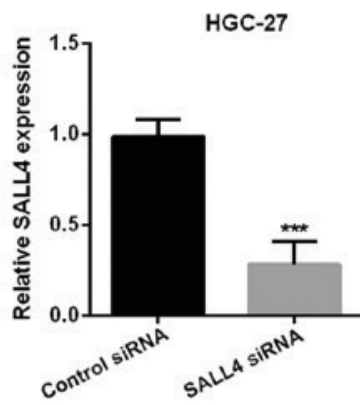

HGC-27
C

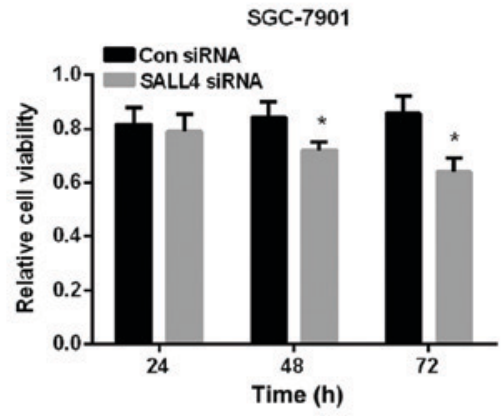

D

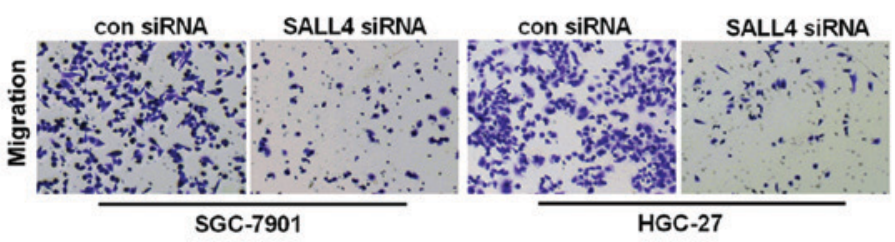

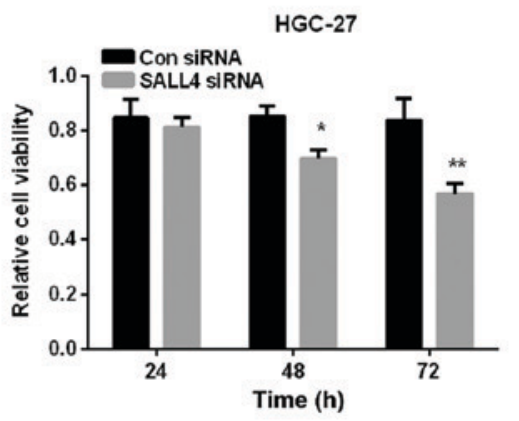
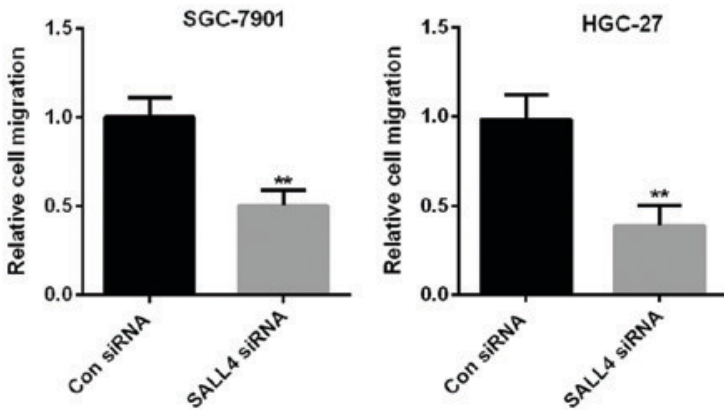

Figure 3. Inhibitory effect of SALL4 siRNA on GC cell proliferation and migration. (A) SALL4 protein content in SGC-7901 and HGC-27 cell lines after downregulation SALL4. (B) SALL4 mRNA content in SGC-7901 and HGC-27 cell lines after downregulation of SALL4. (C) GC cell viability in two cell lines examined by MTT assay after silencing SALL4. (D) GC cell migration in the cell lines examined by Transwell assay after silencing SALL4. "P $<0.05$, ${ }^{* *} \mathrm{P}<0.01,{ }^{* * * *} \mathrm{P}<0.001$.

(cat. no. sc-2004; 1:3,000; Santa Cruz Biotechnology, Inc. Santa Cruz, CA, USA) were added and incubated at room temperature for $2 \mathrm{~h}$. Finally, the enhanced chemiluminescence kit (ECL; EMD Millipore, Billerica, MA, USA) was used to detect the signals. GAPDH primary antibody (cat. no. 70699; 1:5,000; Abcam, Cambridge, MA, USA) was chosen as the internal reference.

Dual-luciferase assay. The wild-type and mut-type miR-16 putative targets on SALL4 3'UTR were synthesized and inserted into the pMIR-reporter luciferase vector. We used Lipofectamine 2000 (Invitrogen; Thermo Fisher Scientific Inc.) to transfect HGC-27 cells with control mimic and miR-16 mimic. The One-Glo luciferase assay instrument (Promega Corporation, Madison, WI, USA) was then used to measure the luciferase activity values.

Statistical analysis. All results are presented as the mean \pm SD of three experiments. Differences between groups were evaluated by Student's t-test or Tukey's post hoc test after ANOVA in SPSS. The difference between groups was significant at P-value <0.05. SPSS v.19.0 software (SPSS, Inc., Chicago, IL, USA) was used to perform statistical analyses and GraphPad Prism 5.02 (GraphPad Software, Inc., La Jolla, CA, USA) to complete graph presentation.

\section{Results}

Increase of miR-16 and decrease of CRKL in GC. First, we examined miR-16 expression in forty pairs of GC tissues. RT-qPCR showed that miR-16 average expression was markedly decreased in GC tissues (Fig. 1A). Next, we assessed the correlation of miR-16 expression level and the stage of cancer. The results indicated that miR-16 showed higher expression in stage I/II (early stage) GC tissues than in stage III/IV (late stage) (Fig. 1B). Subsequently, we detected miR-16 mRNA expression in GC cell lines. Compared with the normal GES-1 cells, miR-16 expression was reduced significantly in GC cell lines (Fig. 1C). Secondly, we examined SALL4 expression in forty pairs of GC tissues and cells by RT-qPCR and immunoblotting respectively. The results showed that SALL4 expression was markedly increased in GC in comparison with normal (Fig. 1D and E), similar results were seen in GC cell lines (Fig. 1F and G).

Inhibition effect of miR-16 on GC cell proliferation and migration. We overexpressed or silenced miR-16 by transfection of 
A

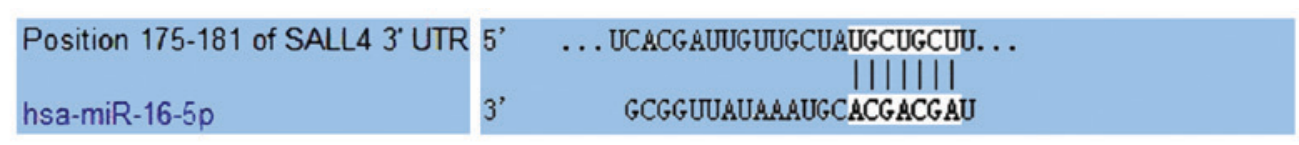

B
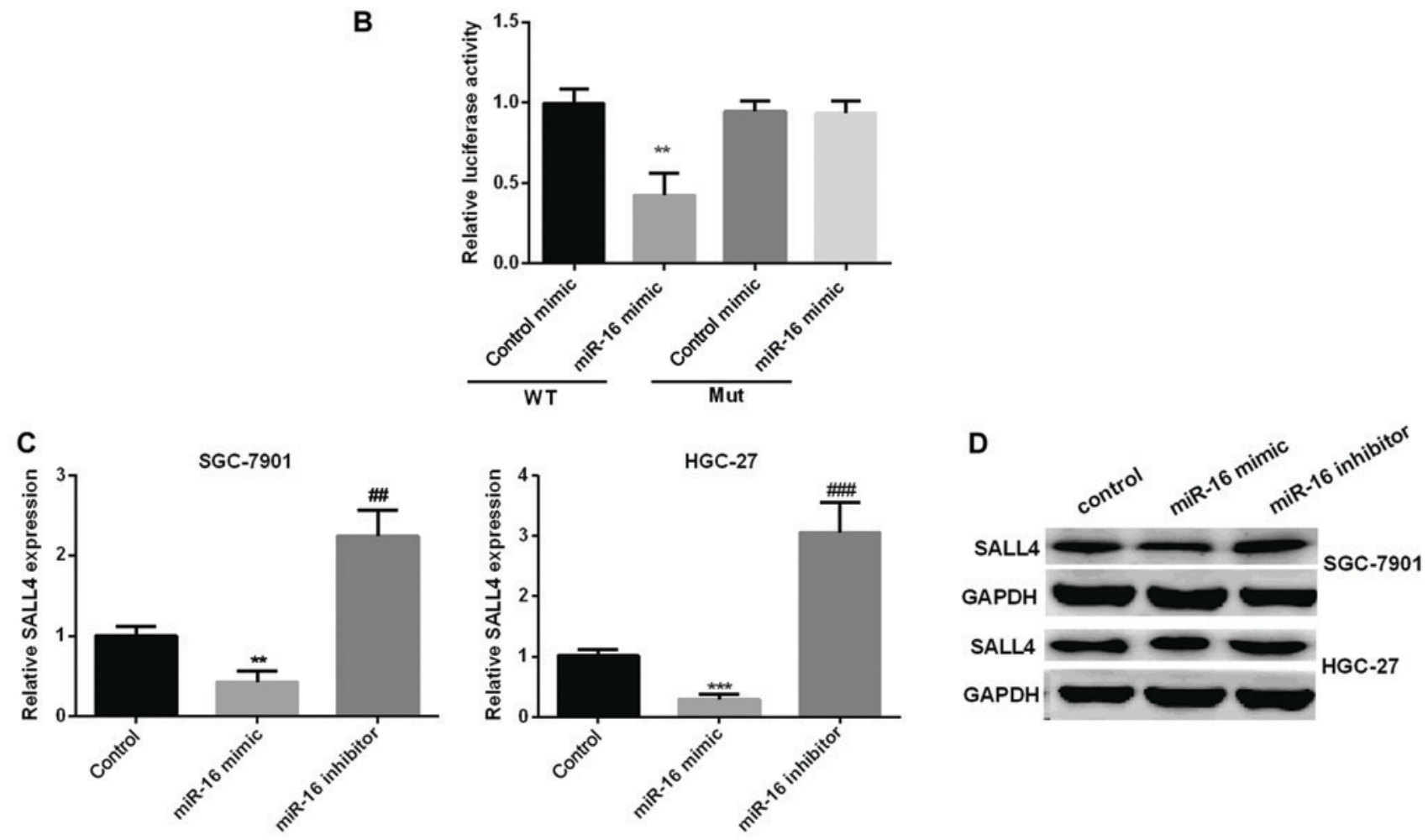

Figure 4. Corroboration of SALL4 as the target of miR-16 in GC. (A) The binding sites of miR-16 with the 3'-UTR of SALL4. (B) Relative luciferase activities in GC cells after treated with miR-16 mimic. (C) Relative SALL4 mRNA expression in the GC cell lines after miR-16 overexpression or silence. (D) Relative SALL4 protein expression in the GC cell lines after miR-16 overexpression or silence. ${ }^{* *} \mathrm{P}<0.01,{ }^{* * *} \mathrm{P}<0.001 ;{ }^{\# \#} \mathrm{P}<0.01,{ }^{\# \# \#} \mathrm{P}<0.001$.

miR-16 mimic or inhibitor into SGC-7901 and HGC-27 cells. The efficiency of the miR-16 transfection was assessed by RT-qPCR and found that miR-16 expression was obviously higher in both GC cells after overexpression of miR-16 but was decreased after silencing miR-16 compared with the control (Fig. 2A and B). We used MTT assay to measure miR-16 effect on GC cell proliferation. As Fig. 2C and D show, re-expression of miR-16 made cell viability reduced in both GC cell lines, while, inhibiting miR-16 significantly raised cell viability. Next, we used Transwell assay to examine miR-16's effect on GC cell migration. As seen in Fig. 2E and F, miR-16 re-expression significantly reduced the migration cells in both GC cell lines, whereas, miR-16 silencing increased the migration of cells remarkably.

SALL4 silencing inhibits GC cell viability and migration. SALL4 siRNA was performed to knock down SALL4 expression to examine SALL4 function in GC progression. Relative SALL4 expression was detected by western blot analysis and RT-qPCR in SGC-7901 and HGC-27 cell lines after transfected with small interfering RNA, respectively, as shown in Fig. 3A and B, the corresponding SALL4 protein expression and mRNA expression was significantly reduced by downregulation of SALL4 in SGC-7901 and HGC-27 cell lines. Then, we used MTT assay to investigate the cell viability in GC cell lines to explore SALL4 effect on GC cell proliferation. We found that si-SALL4 suppressed cell viability in both GC cell lines (Fig. 3C). Transwell assay revealed that si-SALL4 curbed cell migration also in the GC cell lines (Fig. 3D).

SALL4 is a specific target of miR-16 in GC development. We used TargetScan algorithms to look for possible targets of miR-16. Based on its important role in the process of cell proliferation and migration, we selected SALL4 for further study. To corroborate the hypothesis that SALL4 was a novel target of miR-16 in GC progression, dual-luciferase reporter assay was carried out to check the luciferase activity of HGC-27 cells treated with miR-16 mimic. The results indicated that miR-16 mimic significantly reduced the relative SALL4 luciferase activity in wild-type, however, there were no changed in mut-type (Fig. 4A and B). We then explored the connection between miR-16 and SALL4 expression. RT-qPCR and immunoblotting were carried out to detect SALL4 expression in the GC cell lines by re-expression or knockdown of miR-16. As seen in Fig. 4C and D, the relative SALL4 mRNA and protein expression was reduced observably in miR-16 mimic group, while increased in miR-16 inhibitor group.

The reversal of SALL4 in miR-16 suppression effect in GC. We carried out MTT and Transwell assay to examine SALL4 function in GC cell proliferation and migration regulated by miR-16. As proved above, the miR-16 mimic group showed decreased cell viability. However, re-expression of both miR-16 and 
A

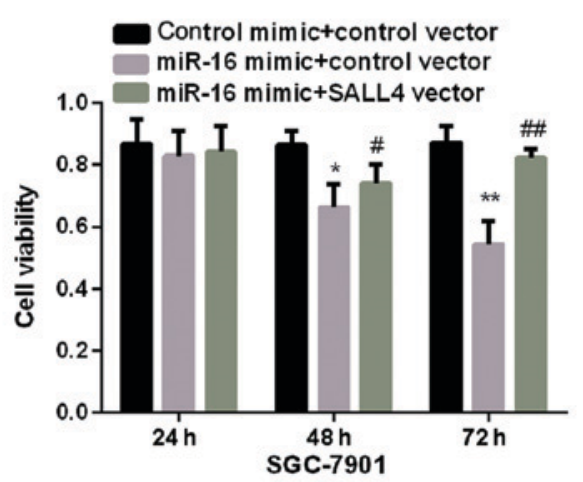

C

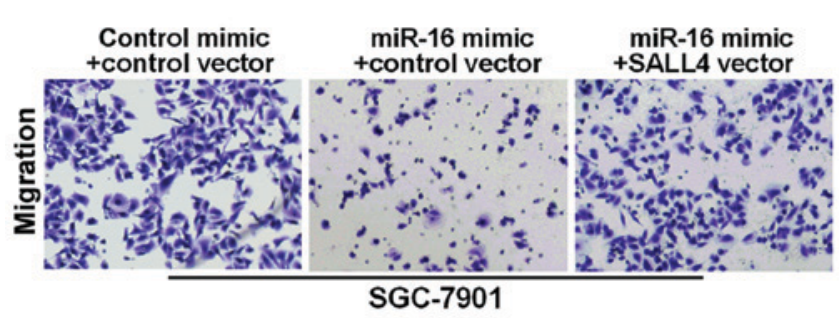

D

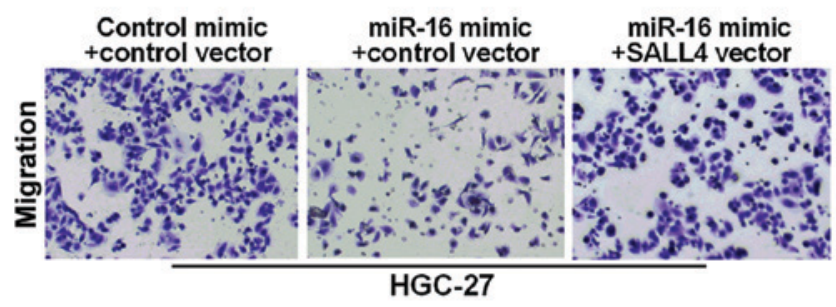

B

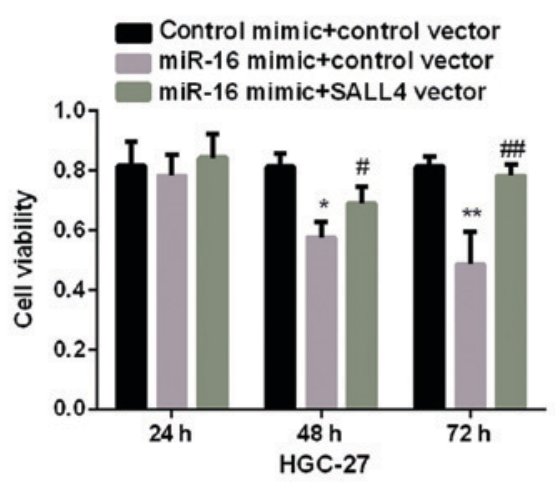

SGC-7901

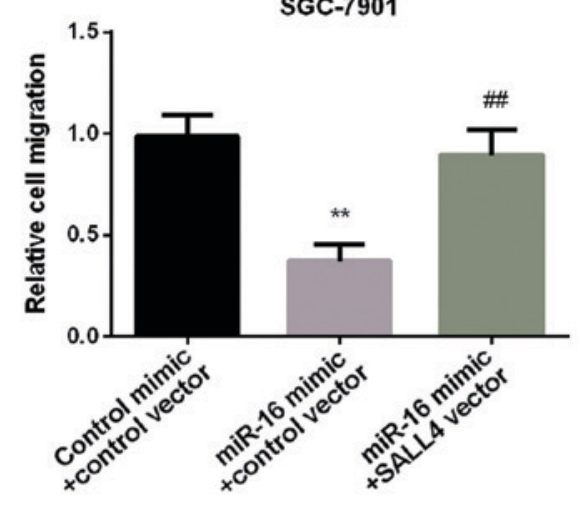

HGC-27

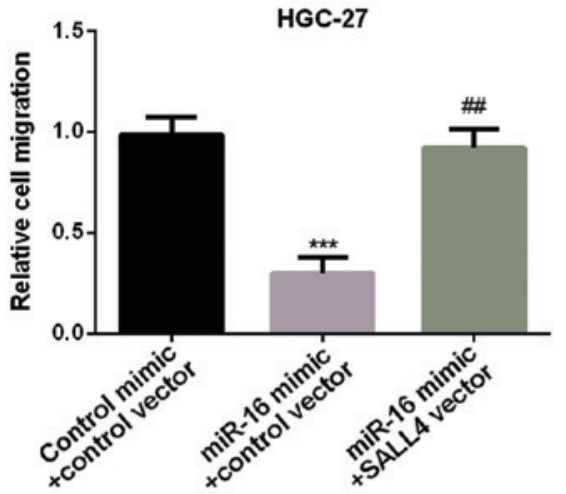

Figure 5. Reversal effect of SALL4 on GC cell proliferation and migration regulated by miR-16. (A and B) Quantitative cell viability in GC cells treated with both miR-16 mimic and SALL4 vector or miR-16 mimic by MTT assays. (C and D) Image showcasing and quantitative cell migration in GC cells after treatment with both miR-16 mimic and SALL4 vector or miR-16 mimic alone by Transwell migration assays. ${ }^{*} \mathrm{P}<0.05,{ }^{* *} \mathrm{P}<0.01,{ }^{* * *} \mathrm{P}<0.001 ;{ }^{\#} \mathrm{P}<0.05,{ }^{\# \#} \mathrm{P}<0.01$.

SALL4 showed higher cell viability than cell overexpression of miR-16 alone (Fig. 5A and B), suggesting that SALL4 attenuated the inhibition effect of miR-16 on GC cell proliferation. In addition, Fig. 5C and D results showed that the relative cell migration in GC cells was decreased in miR-16 mimic group. However, re-expression of both miR-16 and SALL4 showed higher migration than cell overexpression of miR-16 alone, suggesting that SALL4 attenuated miR-16 inhibition effect on GC cell migration. In conclusion, miR-16 could inhibit GC cell proliferation and migration by targeting SALL4.

\section{Discussion}

Previous studies have shown that expression of several miRNAs was abnormal in gastric cancer (GC) which in turn induced the changed cell proliferation, invasion and apoptosis (27). Thus, these miRNAs could be used as biomarkers to predict the prognosis of GC and search for a specific miRNA and its target gene is critical.

miR-16 had been proved to be expressed abnormally in a variety of human cancers. It was reported that miR-16 was obviously reduced in pituitary tumors (28). Moreover, miR-16 was reported to be decreased in chronic lymphocytic leukemia cells and targeted Bcl-2 to induce cell apoptosis (29). Recently a study showed that miR-16 was expressed abnormally in GC development and progression $(3,30)$. Our study stated an observably reduced miR-16 expression in GC, and miR-16 mimic suppressed GC cell proliferation and migration, while miR-127 inhibitor facilitated it. It was in line with the recent studies that miR-16 was downregulated in GC and it could inhibit GC cell progression $(31,32)$.

SALL4 is well known to be involved in progression of many human cancers, including colorectal cancer, breast cancer, liver cancer and lung cancer (20,33-35), by regulating cell growth, 
metastasis and invasion. SALL4 expression detected in our experiment was obviously higher in GC consistent with reports that SALL4 was upregulated in GC $(24,36)$. A previous study also showed that SALL4 promoted cell proliferation and metastasis regulated by the miR-33b, and miR-33b exhibited significant inverse correlation with SALL4 in hepatocellular carcinoma cells (37). Zhou et al (38) found that SALL4 expression was directly regulated by miR-16 in glioma cell proliferation, migration and invasion. Our present study indicated that SALL4 expression increased in GC and silencing SALL4 could inhibit GC cell viability and migratory ability.

Colletively, miR-16 expression was downregulated while SALL4 was upregulated in GC. The relationship between miR-16 and SALL4 expression was negatively correlated. We first proved that SALL4 was a directly target of miR-16 in regulation of the progress of GC and SALL4 could partially reverse the suppression effect of miR-16 in GC, indicating miR-16/SALL4 axis to have a potential application vlue in GC diagnosis and therapy.

\section{Acknowledgements}

Not applicable.

\section{Funding}

No funding was received.

\section{Availability of data and materials}

The datasets used and/or analyzed during the present study are available from the corresponding author on reasonable request.

\section{Authors' contributions}

XJ collected and analyzed the data, interpreted the data and drafted the manuscript. ZW conceived and designed this study, finally revised and approved the manuscript. Both authors read and approved the final manuscript.

\section{Ethics approval and consent to participate}

The study was approved by the Ethics Committee of ChinaJapan Union Hospital, Jilin University (Changchun, China). Signed informed consents were obtained from the patients or the guardians.

\section{Patient consent for publication}

Not applicable.

\section{Competing interests}

The authors declare that they have no competing interests.

\section{References}

1. Ferro A, Peleteiro B, Malvezzi M, Bosetti C, Bertuccio P, Levi F, Negri E, La Vecchia C and Lunet N: Worldwide trends in gastric cancer mortality (1980-2011), with predictions to 2015, and incidence by subtype. Eur J Cancer 50: 1330-1344, 2014.
2. Zheng L, Jiao W, Song H, Qu H, Li D, Mei H, Chen Y, Yang F, Li H, Huang K, et al: miRNA-558 promotes gastric cancer progression through attenuating Smad4-mediated repression of heparanase expression. Cell Death Dis 7: e2382, 2016.

3. Zhang J, Song Y, Zhang C, Zhi X, Fu H, Ma Y, Chen Y, Pan F, Wang K, Ni J, et al: Circulating miR-16-5p and miR-19b-3p as two novel potential biomarkers to indicate progression of gastric cancer. Theranostics 5: 733-745, 2015.

4. Tsai MM, Wang CS, Tsai CY, Huang HW, Chi HC, Lin YH, $\mathrm{Lu} \mathrm{PH}$ and Lin KH: Potential diagnostic, prognostic and therapeutic targets of microRNAs in human gastric cancer. Int J Mol Sci 17: 945, 2016.

5. An Y, Zhang Z, Shang Y, Jiang X, Dong J, Yu P, Nie Y and Zhao Q: miR-23b-3p regulates the chemoresistance of gastric cancer cells by targeting ATG12 and HMGB2. Cell Death Dis 6: e1766, 2015.

6. Hurst DR, Edmonds MD and Welch DR: Metastamir: The field of metastasis-regulatory microRNA is spreading. Cancer Res 69: 7495-7498, 2009.

7. Ahn SM, Cha JY, Kim J, Kim D, Trang HT, Kim YM, Cho YH, Park D and Hong S: Smad3 regulates E-cadherin via miRNA-200 pathway. Oncogene 31: 3051-3059, 2012.

8. Zuo QF, Cao LY, Yu T, Gong L, Wang LN, Zhao YL, Xiao B and Zou QM: MicroRNA-22 inhibits tumor growth and metastasis in gastric cancer by directly targeting MMP14 and Snail. Cell Death Dis 6: e2000, 2015.

9. Zhan XH, Xu QY, Tian R, Yan H, Zhang M, Wu J, Wang W and He J: MicroRNA16 regulates glioma cell proliferation, apoptosis and invasion by targeting Wip1-ATM-p53 feedback loop. Oncotarget 8: 54788-54798, 2017.

10. Wang W, Chen J, Dai J, Zhang B, Wang F and Sun Y: MicroRNA-16-1 inhibits tumor cell proliferation and induces apoptosis in A549 non-small cell lung carcinoma cells. Oncol Res 24: 345-351, 2016.

11. Diamantopoulos MA, Kontos CK, Kerimis D, Papadopoulos IN and Scorilas A: Upregulated miR-16 expression is an independent indicator of relapse and poor overall survival of colorectal adenocarcinoma patients. Clin Chem Lab Med 55: 737-747, 2017.

12. Tatetsu H, Kong NR, Chong G, Amabile G, Tenen DG and Chai L: SALL4, the missing link between stem cells, development and cancer. Gene 584: 111-119, 2016.

13. Warren M, Wang W, Spiden S, Chen-Murchie D, Tannahill D, Steel KP and Bradley A: A Sall4 mutant mouse model useful for studying the role of Sall4 in early embryonic development and organogenesis. Genesis 45: 51-58, 2007.

14. Wang J, Rao S, Chu J, Shen X, Levasseur DN, Theunissen TW and Orkin SH: A protein interaction network for pluripotency of embryonic stem cells. Nature 444: 364-368, 2006.

15. Zhang J, Tam WL, Tong GQ, Wu Q, Chan HY, Soh BS, Lou Y, Yang J, Ma Y, Chai L, et al: Sall4 modulates embryonic stem cell pluripotency and early embryonic development by the transcriptional regulation of Pou5f1. Nat Cell Biol 8: 1114-1123, 2006.

16. Wu Q, Chen X, Zhang J, Loh YH, Low TY, Zhang W, Zhang W, Sze SK, Lim B and Ng HH: Sall4 interacts with Nanog and co-occupies Nanog genomic sites in embryonic stem cells. J Biol Chem 281: 24090-24094, 2006.

17. Oikawa T, Kamiya A, Zeniya M, Chikada H, Hyuck AD, Yamazaki Y, Wauthier E, Tajiri H, Miller LD, Wang XW, et al: Sal-like protein 4 (SALL4), a stem cell biomarker in liver cancers. Hepatology 57: 1469-1483, 2013.

18. Kobayashi D, Kuribayashi K, Tanaka M and Watanabe N: Overexpression of SALL4 in lung cancer and its importance in cell proliferation. Oncol Rep 26: 965-970, 2011.

19. Kobayashi D, Kuribayshi K, Tanaka M and Watanabe N: SALL4 is essential for cancer cell proliferation and is overexpressed at early clinical stages in breast cancer. Int J Oncol 38: 933-939, 2011.

20. Ardalan Khales S, Abbaszadegan MR, Abdollahi A, Raeisossadati R, Tousi MF and Forghanifard MM: SALL4 as a new biomarker for early colorectal cancers. J Cancer Res Clin Oncol 141: 229-235, 2015.

21. Itou J, Matsumoto Y, Yoshikawa K and Toi M: Sal-like 4 (SALL4) suppresses $\mathrm{CDH} 1$ expression and maintains cell dispersion in basal-like breast cancer. FEBS Lett 587: 3115-3121, 2013.

22. Li A, Jiao Y, Yong KJ, Wang F, Gao C, Yan B, Srivastava S, Lim GS, Tang P, Yang H, et al: SALL4 is a new target in endometrial cancer. Oncogene 34: 63-72, 2015.

23. Zhang X, Yuan X, Zhu W, Qian H and Xu W: SALL4: An emerging cancer biomarker and target. Cancer Lett 357: 55-62, 2015 . 
24. Zhang L, Xu Z, Xu X, Zhang B, Wu H, Wang M, Zhang X, Yang T, Cai J, Yan Y, et al: SALL4, a novel marker for human gastric carcinogenesis and metastasis. Oncogene 33: 5491-5500, 2014.

25. Yuan $X$, Zhang $X$, Zhang W, Liang W, Zhang P, Shi H, Zhang B, Shao M, Yan Y, Qian H, et al: SALL4 promotes gastric cancer progression through activating CD44 expression. Oncogenesis 5: e268, 2016.

26. Livak KJ and Schmittgen TD: Analysis of relative gene expression data using real-time quantitative PCR and the 2(-Delta Delta C(T)) Method. Methods 25: 402-408, 2001.

27. Ishiguro H, Kimura M and Takeyama $H$ : Role of microRNAs in gastric cancer. World J Gastroenterol 20: 5694-5699, 2014.

28. Amaral FC, Torres N, Saggioro F, Neder L, Machado HR, Silva WA Jr, Moreira AC and Castro M: MicroRNAs differentially expressed in ACTH-secreting pituitary tumors. J Clin Endocrinol Metab 94: 320-323, 2009.

29. Cimmino A, Calin GA, Fabbri M, Iorio MV, Ferracin M, Shimizu M, Wojcik SE, Aqeilan RI, Zupo S, Dono M, et al: miR-15 and miR-16 induce apoptosis by targeting BCL2. Proc Natl Acad Sci USA 102: 13944-13949, 2005.

30. Venturutti L, Cordo Russo RI, Rivas MA, Mercogliano MF, Izzo F, Oakley RH, Pereyra MG, De Martino M, Proietti CJ, Yankilevich P, et al: MiR-16 mediates trastuzumab and lapatinib response in ErbB-2-positive breast and gastric cancer via its novel targets CCNJ and FUBP1. Oncogene 35: 6189-6202, 2016.

31. Kang W, Tong JH, Lung RW, Dong Y, Zhao J, Liang Q, Zhang L, Pan Y, Yang W, Pang JC, et al: Targeting of YAP1 by microRNA-15a and microRNA-16-1 exerts tumor suppressor function in gastric adenocarcinoma. Mol Cancer 14: 52, 2015.

32. Wang T, Hou J, Li Z, Zheng Z, Wei J, Song D, Hu T, Wu Q, Yang JY and Cai JC: miR-15a-3p and miR-16-1-3p negatively regulate Twist 1 to repress gastric cancer cell invasion and metastasis. Int J Biol Sci 13: 122-134, 2017.
33. Gautam AK, Wang C, Zeng J, Wang J, Lu J, Wei J, Huang G, Mo B, Luo M and Mo B: Expression and clinical significance of SALL4 and LGR5 in patients with lung cancer. Oncol Lett 10: 3629-3634, 2015.

34. Tanaka Y, Aishima S, Kohashi K, Okumura Y, Wang H, Hida T, Kotoh K, Shirabe K, Maehara Y, Takayanagi R, et al: Spalt-like transcription factor 4 immunopositivity is associated with epithelial cell adhesion molecule expression in combined hepatocellular carcinoma and cholangiocarcinoma. Histopathology 68: 693-701, 2016.

35. Dirican E and Akkiprik M: Functional and clinical significance of SALL4 in breast cancer. Tumour Biol 37: 11701-11709, 2016.

36. Liu J, Wang L, Yang A, Jiang P and Wang M: Up-regulation of SALL4 associated with poor prognosis in gastric cancer. Hepatogastroenterology 61: 1459-1464, 2014.

37. Tian Q, Xiao Y, Wu Y, Liu Y, Song Z, Gao W, Zhang J, Yang J, Zhang Y, Guo T, et al: MicroRNA-33b suppresses the proliferation and metastasis of hepatocellular carcinoma cells through the inhibition of Sal-like protein 4 expression. Int J Mol Med 38: 1587-1595, 2016.

38. Zhou Y, Liu Y, Hu C and Jiang Y: MicroRNA-16 inhibits the proliferation, migration and invasion of glioma cells by targeting Sal-like protein 4. Int J Mol Med 38: 1768-1776, 2016.

(1) (9) This work is licensed under a Creative Commons Attribution-NonCommercial-NoDerivatives 4.0 International (CC BY-NC-ND 4.0) License. 